OPEN ACCESS

Edited by:

Angel Gil-Izquierdo, Spanish National Research

Council, Spain

Reviewed by:

Mar Larrosa,

European University of Madrid, Spain

Venkatesan Arul,

Pondicherry University, India

*Correspondence:

Shuiyuan Cheng

s_y_cheng@sina.com

Zhenzhou Zhu

zhenzhou.zhu@whpu.edu.cn

Francisco J. Barba

francisco.barba@uv.es

Specialty section:

This article was submitted to

Nutrition and Food Science

Technology,

a section of the journal

Frontiers in Nutrition

Received: 18 March 2020

Accepted: 21 August 2020 Published: 05 November 2020

Citation:

Chen X, Li S, Cong X, Yu T, Zhu Z, Barba FJ, Marszalek K, Puchalski C and Cheng S (2020) Optimization of Bacillus cereus Fermentation Process for Selenium Enrichment as Organic

Selenium Source.

Front. Nutr. 7:543873.

doi: 10.3389/fnut.2020.543873

\section{Optimization of Bacillus cereus Fermentation Process for Selenium Enrichment as Organic Selenium Source}

\author{
Xujun Chen ${ }^{1}$, Shuyi $\mathrm{Li}^{1}$, Xin Cong ${ }^{1,2}$, Tian Yu ${ }^{1,2}$, Zhenzhou Zhu ${ }^{1 *}$, Francisco J. Barba ${ }^{3 *}$, \\ Krystian Marszalek ${ }^{4,5}$, Czesław Puchalski ${ }^{6}$ and Shuiyuan Cheng ${ }^{1 *}$ \\ ${ }^{1}$ National R\&D Center for Se-rich Agricultural Products Processing, College of Food Science and Engineering, Wuhan \\ Polytechnic University, Wuhan, China, ${ }^{2}$ Enshi Se-Run Health Tech Development Co., Ltd, Enshi City, China, ${ }^{3}$ Nutrition and \\ Food Science Area, Department of Preventive Medicine and Public Health, Food Science, Toxicology and Forensic Medicine, \\ Faculty of Pharmacy, Universitat de València, Avda. Vicent Andrés Estellés, València, Spain, ${ }^{4}$ Department of Fruit and \\ Vegetable Product Technology, Prof. Wacław Dabrowski Institute of Agricultural and Food Biotechnology, Warsaw, Poland, \\ ${ }^{5}$ Department of Chemistry and Food Toxicology, Institute of Food Technology and Nutrition, College of Natural Sciences, \\ University of Rzeszów, Rzeszow, Poland, ${ }^{6}$ Department of Bioenergetics, Food Analysis and Microbiology, Institute of Food \\ Technology and Nutrition, College of Natural Sciences, University of Rzeszów, Rzeszow, Poland
}

Selenium is an essential trace element and micronutrient for human health. Application of organic selenium in plants and microorganisms as trace element supplement is attracting more and more attention. In this study, Bacillus cereus, an important probiotic, was used for selenium enrichment with sodium selenite as selenium source. The growth curve of $B$. cereus was investigated, and $150 \mu \mathrm{g} / \mathrm{ml}$ was selected as the concentration of selenium for $B$. cereus fermentation. With application of response surface methodology, the optimal fermentation conditions were obtained as follows: inoculation quantity of $7 \%$, culture temperature of $33^{\circ} \mathrm{C}$, and shaking speed of $170 \mathrm{rpm}$, leading to the maximal selenium conversion ratio of $94.3 \pm 0.2 \%$. Field emission scanning electron microscope and energy dispersive spectrometry evidenced that inorganic selenium had been successfully transformed. This study may contribute to get a strain with high Se conversion ratio, so as to extract organic selenium in the form of selenoprotein to be used for further application.

Keywords: selenium enrichment, Bacillus cereus, fermentation, response surface methodology, organic selenium

\section{INTRODUCTION}

As an essential trace element, as well as a kind of micronutrient, selenium (Se) was first discovered in 1817 by Swedish scientists in the sludge of leaden chambers used for production of sulfuric acid. Selenium plays a key role in maintaining the balance of human immune, endocrine, metabolism, and intracellular environment (1). Especially in the immune system, selenium can bind to erythrocyte, albumin, and plasma globulin and be transported to tissues after being absorbed by organisms, and 28 to $46 \%$ of selenium can deposit in skeletal muscle (2). 
Selenium is also an important component of the glutathione peroxidase (GSH-px) subunit, which can detoxify free radicals and protect the body from injury (3). Deficiency of selenium in humans can lead to a series of adverse health conditions, such as Keshan disease, Kashin-Beck disease, poorer immune function, and problematic fertility/reproduction (4). Selenium can be roughly divided into inorganic selenium and organic selenium. Inorganic selenium is mainly composed of selenate and selenite with the characteristics of low bioavailability and toxicity when in excess (5), whereas organic selenium contains a variety of selenium amino acids (such as selenomethionine and selenocysteine) (6), and it can provide antioxidant benefits by acting both as direct antioxidants and as a source of selenium for synthesis of selenium-dependent antioxidant and repair of proteins (e.g., glutathione peroxidases, thioredoxin reductases, and methionine sulfoxide reductases) (7). In recent years, researches on biological transformation of selenium have begun to attract much attention. Two methods of bioconversion of selenium have been reported: microbial biotransformation and selenium-enriched sprouted seeds via sprouting (8). Fermentation of cultures of yeasts and lactic acid bacteria for selenium enrichment have been studied a lot recently, showing that bioconversion by microorganisms was faster and safer than selenium-enriched sprouted seeds.

Previous studies have shown that some bacteria can convert inorganic selenium into organic selenium in the form of protein (9). Hong et al. (10) studied the effects of different selenium concentrations on the growth of Fusarium spp., and results showed that adding selenium within the concentration of 0.10 $1.00 \mathrm{mg} / \mathrm{L}$ could evidently promote the mycelial growth of Fusarium tricinctum. Yuan et al. (11) optimized the medium formulation of beer yeast by single factor and orthogonal test and prepared selenium-enriched beer yeast with a total selenium content of $23 \mathrm{mg} / \mathrm{L}$. Low selenium concentration can affect the growth of Streptococcus acidophilus bacteria, the bioaccumulation of selenium and the form of selenium. By increasing the concentration of sodium selenite, the dry weight of $S$. acidophilus can be significantly increased. At the same time, selenium can bind to bacterial proteins and form selenocysteine (12). Bacillus cereus, an endospore-forming, grampositive bacterium, is of great economic importance due to its ability to produce various enzymes, such as amylase (13), protease (14), and cellulase (15). As probiotics, B. cereus can produce many beneficial effects for organisms. Using $B$. cereus as a feed additive can affect the intestinal immune system of piglets after feeding and enhance the double-positive cell population of $\mathrm{cd} 8 / \mathrm{cd} 3$ in the intestinal epithelium of a probiotics group (16). However, to our best knowledge, selenium enrichment in B. cereus via fermentation has been barely studied.

The purpose of this study was to investigate the selenium enrichment ability of $B$. cereus. The effect of selenium concentration in the form of sodium selenite on selenium transforming during $B$. cereus fermentation was studied. The fermentation parameters such as inoculation quantity, culture temperature, and shaking speed were optimized to obtain the optimal selenium conversion ratio. The surface characterization of selenium-enriched $B$. cereus was carried out using field emission scanning electron microscope and energy dispersive spectrometry.

\section{MATERIALS AND METHODS}

\section{Strain Material}

The experimental B. cereus strains were isolated from soil that was taken from Enshi (China) and obtained by Se-Run Health Tech Development Co., Ltd., in Enshi (China). The phylogenetic tree of the strain obtained from $16 \mathrm{~S}$ rDNA sequencing analysis showed that the homology of the strain with $B$. cereus strain reached $99 \%$. The strain was collected at $-80^{\circ} \mathrm{C}$ and inoculated into Luria-Bertani (LB) agar medium plates and activated at $37^{\circ} \mathrm{C}$ for $6 \mathrm{~h}$, and then single colonies were selected from it and inoculated into liquid LB medium. The strain seed liquid was obtained by culture at $150 \mathrm{rpm}$ on a shaking incubator HZ150L (Ruihua Instrument \& Equipment, China) with a rotational radius of $20 \mathrm{~mm}$ (same rotational radius below) for $12 \mathrm{~h}$, and the concentration is $3 \times 10^{7} \mathrm{cfu} / \mathrm{ml}$.

\section{Chemicals and Reagents}

The bacterial genomic DNA extraction kit was purchased from Biovision (Milpitas, California, USA). The Gel Extraction Kit was purchased from Qiagen (Germany). The DNA agarose gel Recovery Kit was purchased from Solarbio (Beijing, China). Analytical grade sodium selenite, analytical grade hydrochloric acid sodium chloride, and ethylenediaminetetraacetic acid disodium salt (EDTA-2Na) were purchased from Sinopharm Chemical Reagent Co., Ltd. (Shanghai, China). O-phenylenediamine was purchased from Aladdin Biochemical Technology Co., Ltd. (Shanghai, China). Tryptone was purchased from OXOID (Hants, UK). Yeast extract was purchased from Aoboxing Biotechnology (Beijing, China). Agar was purchased from Biofroxx (Germany).

\section{Determination of Strain Growth Curve}

Strain seed liquid was inoculated into $50 \mathrm{ml}$ liquid LB medium with $5 \%$ inoculation amount and cultivated at $37^{\circ} \mathrm{C}$ and $150 \mathrm{rpm}$. The absorbance of $B$. cereus at $600 \mathrm{~nm}$ was measured per $1 \mathrm{~h}$ by the growth curve tester Bioscreen C (Finland) to obtain the growth curve.

\section{Optimization of $B$. cereus Fermentation for Selenium Enrichment Optimization of Fermentation Conditions for Selenium Enrichment}

Sodium selenite $\left(\mathrm{Na}_{2} \mathrm{SeO}_{3}\right)$, source of selenium, was dissolved in distilled water $(10 \mathrm{mg} / \mathrm{L})$ and sterilized by high-pressure steam. Strain seed liquid was inoculated into LB liquid medium with $5 \%$ inoculation volume (total volume $50 \mathrm{ml}$ ), and sodium selenite was added during logarithmic growth period (4h) with the concentration of $150 \mu \mathrm{g} / \mathrm{ml}$, according to previous tests to assure sufficient source selenium content. The fermentation duration was $24 \mathrm{~h}$. On the basis of the single factor tests, Box-behnken experimental design was used to optimize the fermentation conditions. The strain seed liquid was inoculated into LB liquid medium with an inoculation amount of $1 \sim 9 \%$, and then culture 
TABLE 1 | The code levels of investigated parameters.

\begin{tabular}{lccc}
\hline Variables & \multicolumn{3}{c}{ Code level } \\
\cline { 2 - 4 } & $-\mathbf{- 1}$ & $\mathbf{0}$ & $\mathbf{1}$ \\
\hline Inoculation amount $\left(X_{1}\right), \%$ & 5 & 7 & 9 \\
Culture temperature $\left(X_{2}\right),{ }^{\circ} \mathrm{C}$ & 31 & 33 & 35 \\
Shaking speed $\left(X_{3}\right), \mathrm{rpm}$ & 150 & 170 & 190 \\
\hline
\end{tabular}

TABLE 2 | Response surface analysis test design and results of selenium conversion ratio.

\begin{tabular}{|c|c|c|c|c|c|c|c|}
\hline \multirow[t]{2}{*}{ Run } & \multicolumn{3}{|c|}{ Coded variables } & \multicolumn{3}{|c|}{ Actual variables } & \multirow{2}{*}{$\begin{array}{c}\begin{array}{c}\text { Selenium conversion } \\
\text { ratio }(\%)\end{array} \\
Y\end{array}$} \\
\hline & $A$ & $B$ & $c$ & $X_{1}$ & $X_{2}$ & $X_{3}$ & \\
\hline 1 & 1 & 1 & 0 & 9 & 35 & 170 & 0.705 \\
\hline 2 & 0 & 1 & 1 & 7 & 35 & 190 & 0.720 \\
\hline 3 & 1 & 0 & -1 & 9 & 33 & 150 & 0.797 \\
\hline 4 & 1 & -1 & 0 & 9 & 31 & 170 & 0.651 \\
\hline 5 & -1 & 0 & -1 & 5 & 33 & 150 & 0.814 \\
\hline 6 & -1 & -1 & 0 & 5 & 31 & 170 & 0.702 \\
\hline 7 & 0 & 1 & -1 & 7 & 35 & 150 & 0.717 \\
\hline 8 & 0 & 0 & 0 & 7 & 33 & 170 & 0.962 \\
\hline 9 & 0 & -1 & 1 & 7 & 31 & 190 & 0.640 \\
\hline 10 & 0 & 0 & 0 & 7 & 33 & 170 & 0.927 \\
\hline 11 & 0 & 0 & 0 & 7 & 33 & 170 & 0.905 \\
\hline 12 & 0 & 0 & 0 & 7 & 33 & 170 & 0.908 \\
\hline 13 & -1 & 0 & 1 & 5 & 33 & 190 & 0.769 \\
\hline 14 & -1 & 1 & 0 & 5 & 35 & 170 & 0.732 \\
\hline 15 & 0 & 0 & 0 & 7 & 33 & 170 & 0.906 \\
\hline 16 & 0 & -1 & -1 & 7 & 31 & 150 & 0.701 \\
\hline 17 & 1 & 0 & 1 & 9 & 33 & 190 & 0.722 \\
\hline
\end{tabular}

$A$ and $X_{1}$ represent inoculation amount (\%); $B$ and $X_{2}$ are the culture temperature $\left({ }^{\circ} \mathrm{C}\right.$ ), and $C$ and $X_{3}$ are the shaking speed (rpm).

temperature was set at $29 \sim 37^{\circ} \mathrm{C}$ with shaking speed of $110 \sim 190$ $\mathrm{rpm}$. The coding and actual level of independent variables of the process are shown in Table 1. The experimental runs performed are listed in Table 2.

The selenium conversion ratio $(Y)$ was fitted to a quadratic regression model for response surface analysis, as shown in Equation (1):

$$
\begin{aligned}
Y= & \beta_{0}+\beta_{1} A+\beta_{2} B+\beta_{3} C+\beta_{4} A B+\beta_{5} A C+\beta_{6} B C+\beta_{7} A^{2} \\
& +\beta_{8} B^{2}+\beta_{9} C^{2}
\end{aligned}
$$

Where $A, B$, and $C$ correspond to the coded independent variables, namely, inoculation amount, culture temperature, and shaking speed. The $\beta_{0} \sim \beta_{9}$ values represent the corresponding regression coefficients. The experiments were randomized to maximize the effect of unexplained variability on observed responses due to exogenous factors.

\section{Determination of Selenium Conversion Ratio}

The residual inorganic selenium content in the fermentation solution was analyzed according to Zeng et al. (17). Briefly, the cultured strain liquid was centrifuged for $20 \mathrm{~min}$ at 2,415 g, then the absorbance value of the supernatant was measured at $600 \mathrm{~nm}$ $\left(\mathrm{OD}_{600 \mathrm{~nm}}\right)$ to determine the inorganic selenium content by applying the standard curve equation. The selenium conversion ratio $\left(R_{\mathrm{Se}}\right)$ was calculated as follows:

$$
R_{S e}=\frac{S e_{t}-S e_{i}}{S e_{t}} \times 100 \%
$$

where $\mathrm{Se}_{t}$ is the total selenium content $(\mathrm{mg})$ in $50 \mathrm{ml}$ medium, and $S e_{i}$ is the inorganic selenium content $(\mathrm{mg})$ in $50 \mathrm{ml}$ medium after fermentation.

\section{Surface Element Analysis of Selenium-Enriched $B$. cereus}

The strain liquid after fermentation was centrifuged at 2,415 $\mathrm{g}$ for $20 \mathrm{~min}$, then the supernatant was discarded and the precipitate was washed with $\sim 35 \mathrm{ml}$ sterile water to remove the residue selenium in the solution or adsorbed at the surface of strain. Then the freeze-dried strain powder (with or without selenium enrichment) was pasted on the copper sheet with conductive adhesive and sprayed with gold. Then the surface morphology was analyzed by field emission scanning electron microscope (SEM) (SIGMA 500, Carl Zeiss AG, Germany) under accelerated voltage of $3 \mathrm{kV}$, and the surface elements in the field of vision were qualitatively observed by energy dispersive spectrometry (EDS).

\section{Statistical Analysis}

All trials were carried out in triplicate, and all the data were reported as means $\pm \mathrm{SD}$ (standard deviation). The statistical significance was evaluated using Student's $t$ test, and $p<0.05$ or 0.01 was taken as significant.

\section{RESULTS AND DISCUSSION \\ Determination of Growth Curve}

The growth curve of $B$. cereus is shown in Figure 1. The strain was adapting to the new culture environment in lag phase at $0-4 \mathrm{~h}$, when the increase of absorbance value was not obvious. At $4 \mathrm{~h}$, the bacteria begin to enter a logarithmic growth phase, when the bacteria metabolize vigorously and the transformation rate is high; the absorbance value begins to increase significantly. At $24 \mathrm{~h}$, the absorbance value of bacterial solutions began to decrease, and then the bacteria entered the senescent phases, with a large number of autolysis and low transformation rate. Suhajda et al. found that adding inorganic selenium in prophase of the logarithmic phase resulted in the highest selenium content of yeast cells. Moreover, with the proliferation of cells in the logarithmic phase, Se enrichment ability increased and then gradually weakened as the stable phase approached (18). Jin et al. (19) also found that adding sodium selenite at the initial stage of the logarithmic phase could obtain the maximum selenium content and selenium conversion rate of strain. Congcong et al. studied the effect of different selenium addition time on the total selenium 




FIGURE 1 | The growth curve of strain in culture.

content of yeast and Lactobacillus, and the results showed that adding sodium selenite in prophase of the logarithmic phase could obtain a higher total selenium content than in the prophase of growth stage and the metaphase and anaphase of logarithmic phase (20). Hong et al. (21) found that the organic selenium content and selenium conversion ratio of Enterobacter mori first increased and then decreased with selenium addition time and reached the maximum in the logarithmic growth phase, then decreased in the senescent phases. Therefore, the $4 \mathrm{~h}$ logarithmic growth period was chosen as the time of adding sodium selenite, and the culture time was $24 \mathrm{~h}$.

\section{Optimization of Fermentation Process for Selenium Enrichment}

It can be seen from Table 3 that the overall model $p$ value is $<0.0001$, demonstrating that the regression equation was very significant. The lack of fit value $(p$ value $=0.8266>0.05)$ suggested that it was an adequate model to accurately predict the response variable. The coefficient $R^{2}=0.9836$ also indicated the goodness of the model for selenium conversion ratio. Regression analysis showed that $A^{2}, B^{2}$, and $C^{2}$ have a very significant effect on the selenium conversion ratio $(p<0.01)$, and $A, B$, and $C$ have a significant effect on the protein extraction rate $(p<$ 0.05). Among the three variables, culture temperature was the most significant factor affecting the selenium conversion ratio, according to the $p$ value. The next most significant factor was the shaking speed, followed by the inoculation amount.

Figure 2 shows the 3D surface response plots, representing the complex interaction between fermentation conditions on selenium conversion ratio. When the inoculation amount increased from 1 to $7 \%$, the selenium conversion ratio was augmented from 58 to $83.6 \%$. However, further increase of inoculation amount led to lower selenium conversion ratio. The increase of culture temperature and shaking speed led to the same trend regarding the variation of selenium conversion ratio. This result could be attributed to the fact that when inoculation amount, culture temperature, and shaking speed was beyond a certain range, the growth of strain may be inhibited.

According to the experimental data and model analysis, the maximum selenium conversion ratio of the strain $(92.5 \%)$ could be achieved under the optimal conditions: inoculation amount $6.772 \%$, culture temperature $33.130^{\circ} \mathrm{C}$, and shaking speed $167.263 \mathrm{rpm}$. In order to verify the availability and reliability of the regression model obtained in the response surface test design, the above optimal preparation parameters were used to verify the test. To facilitate the process of the experimental operation, the inoculation amount used was $7 \%$, the culture temperature was $33^{\circ} \mathrm{C}$, and the shaking speed was 170 $\mathrm{rpm}$, and the three measurements were performed in parallel. In the verification experiments, the selenium conversion ratio was 
TABLE 3 | ANOVA analysis of experimental data.

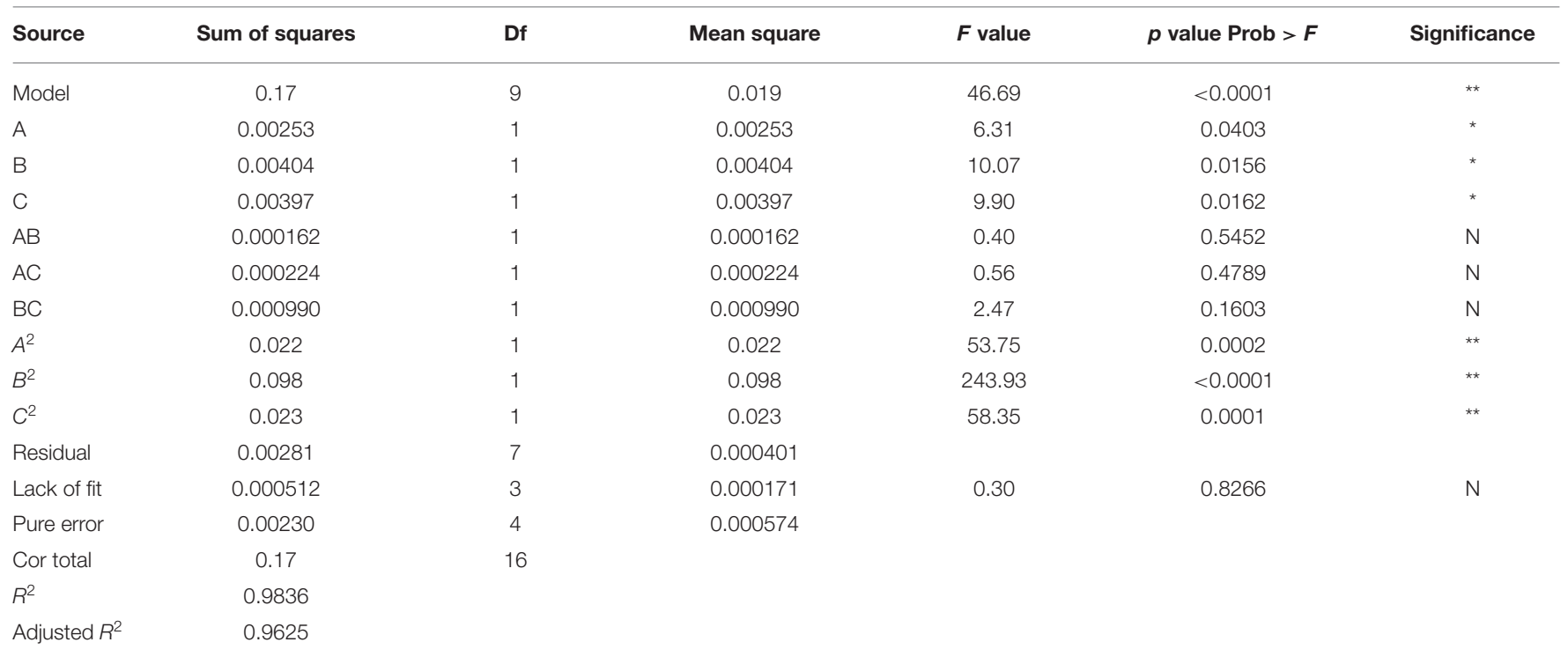

A represents inoculation amount (\%); B represents culture temperature $\left({ }^{\circ} \mathrm{C}\right)$, and $\mathrm{C}$ represents shaking speed (rpm). In the above table, **means very significant difference when $p<$ 0.01 ; * represents the significant difference when $p<0.05$, and $N$ is not significant at $p>0.05$.
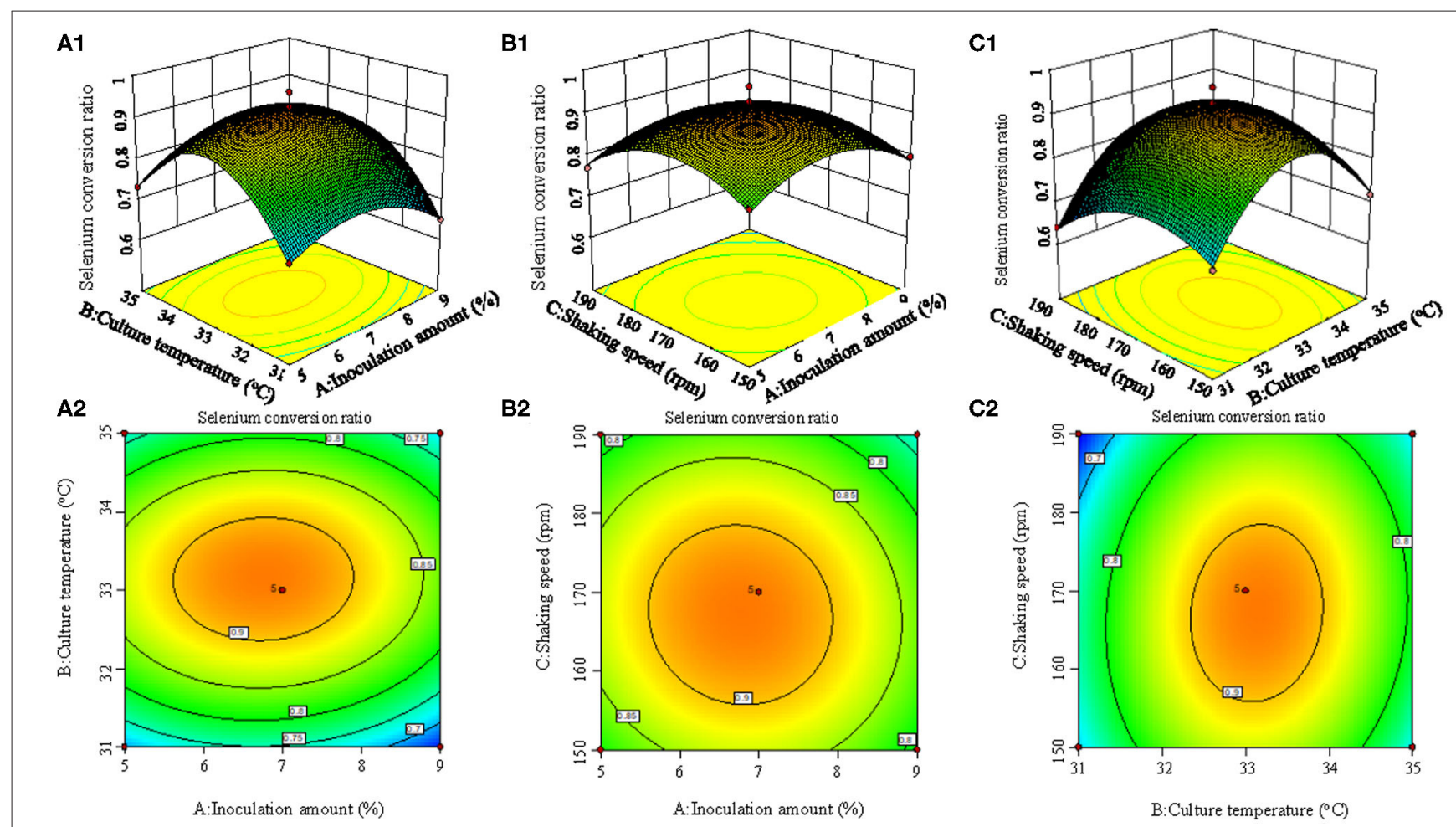

FIGURE 2 | 3D response surface plots (A1, B1, C1) and contour plots (A2, B2, C2) showing the interaction effects of inoculation amount and culture temperature (A1, A2), inoculation amount and shaking speed (B1, B2), and culture temperature and shaking speed (C1, C2) on the selenium conversion ratio.

$94.3 \pm 0.2 \%$, which was close to the predicted value, indicating that the predicted model agrees well with the actual situation. However, the form of organic selenium in the bacteria still needs further characterization.

\section{Surface Element Analysis of Selenium-Enriched Strains}

The surface element analysis of strains with and without selenium enrichment via fermentation was analyzed by energy dispersive 



FIGURE 3 | Element analysis with EDS with (A) and without (B) selenium enrichment.

spectrometry (EDS) (shown in Figure 3). Compared to strains without selenium enrichment, strains after selenium enrichment showed obvious selenium element peak. The intensity of selenium peak at $1.3 \mathrm{keV}$ increased from 0.094 to 0.481 kcps. Energy spectrum analysis provides evidence that the accumulated selenium became the surface material of the strain after fermentation, showing the selenium enrichment ability of $B$. cereus. Yang et al. (22) also found that there were selenium particles on the surface of the selenium-enriched lactic acid bacteria by scanning electron microscopy-energy spectrum analysis. Xu et al. (23) obtained similar results by scanning electron microscopy and energy spectrum analysis of selenium-enriched lactic acid bacteria. Ying et al. (24) observed the selenium-enriched Lactobacillus rhamnosus by transmission electron microscopy and found that there were small black spherical particles with the size of $20-60 \mathrm{~mm}$ on the surface and around the bacteria, which was consistent with the size of elemental selenium, and it was identified as elemental selenium by energy dispersive spectrometry. The same phenomenon was also found in Rhodospirium rubrum, Rhodobacterium sphaeroides, Ralstonia metallifera, and Stenotrophomonas maltophilia (25-28). However, the mechanism needs to be further studied.

\section{CONCLUSIONS}

In this study, B. cereus was cultured for selenium enrichment. The response surface methodology (RSM) was applied to obtain the maximal selenium conversion ratio $(94.3 \pm 0.2 \%)$, under the optimal fermentation parameters: inoculation quantity $7 \%$, culture temperature $33^{\circ} \mathrm{C}$, and shaking speed $170 \mathrm{rpm}$. The energy dispersive spectrometry analysis showed that inorganic selenium in the form of sodium selenite was transformed into organic selenium, as well as $\mathrm{Se}^{0}$ on the surface material of $B$. cereus. This study may contribute to obtain a B. cereus strain with high Se conversion ratio, so as to extract organic selenium in the form of selenoprotein for further work. However, due to the fact that $B$. cereus could generate imbalance in human gut since it can produce bacteriocin that inhibits the growth of other beneficial strains, the selenium-enriched $B$. cereus obtained in this study cannot be used directly as human nutrition supplement like the selenium-enriched yeast and Lactobacillus, leading to further investigation for valorization of organic selenium in B. cereus.

\section{DATA AVAILABILITY STATEMENT}

The raw data supporting the conclusions of this article will be made available by the authors, without undue reservation.

\section{AUTHOR CONTRIBUTIONS}

XCo, TY, and ZZ: conceptualization. XCh and SL: methodology. $\mathrm{XCh}$ : investigation, data curation, and writing-original draft preparation. ZZ: resources. XCh, ZZ, FB, KM, and CP: writingreview and editing. SL and ZZ: supervision. XCo, TY, and SC: project administration. SC: funding acquisition. All authors: have read and agreed to the published version of the manuscript.

\section{FUNDING}

The research was funded by Outstanding Young and Middleaged Science and Technology Innovation Team in Hubei Province (T2020012), Major Technology Innovation of Hubei Province (2019ABA113), Key Research and Development Programme of Hubei Province (2020BBA043), the National youth talent support program in food industry of China, the Scientific and Technology Project of Enshi Tujia and Miao Autonomous Prefecture (D20180004), and the program of the Minister of Science and Higher Education entitled Regional Initiative of Excellence in 2019-2022 (project no. 026/RID/2018/19). 


\section{REFERENCES}

1. Duntas LH, Benvenga S. Selenium: an element for life. Endocrine. (2014) 48:756-75. doi: 10.1007/s12020-014-0477-6

2. Terry EN, Diamond AM. Selenium. Hoboken, NJ: John Wiley \& Sons, Ltd (2012).

3. Huang Y, Ge D-Y, Zon H, Yin J-X, Qu X-N, Lv S-W, editors. Active site mimicry of glutathione peroxidase byglutathione imprinted seleniumcontaining trypsin. Catalysts. (2017) 7:282. doi: 10.3390/catal7100282

4. Rayman MP. Selenium intake, status, and health: a complex relationship. Hormones. (2019) 2019:1-6. doi: 10.1007/s42000-019-00125-5

5. Conde JE, Alaejos MS. Selenium Concentrations in Natural and Environmental Waters. Chem Rev. (1997) 97:19792004. doi: 10.1021/cr960100g

6. Niedzielski P, Rudnicka M, Wachelka M, Kozak L, Rzany M, Wozniak M, et al. Selenium species in selenium fortified dietary supplements. Food Chem. (2016) 190:454-9. doi: 10.1016/j.foodchem.2015.05.125

7. Rahmanto AS, Davies MJ. Selenium-containing amino acids as direct and indirect antioxidants. IUBMB Life. (2012) 64:863-71. doi: 10.1002/iub.1084

8. Diowksz A, Kordialik-Bogacka E, Ambroziak W. Se-enriched sprouted seeds as functional additives in sourdough fermentation. Food Science Technol. (2014) 56:524-8. doi: 10.1016/j.lwt.2013.11.023

9. Sun NX, Liu YH, Wang YX. Utilization of spent brewer's yeast for selenium-enriched yeast. Sustain Dev. (2015) 2016: 781-88. doi: 10.1142/9789814749916_0082

10. Hong Y, Yan Z, Feng Z, Tao HJ, Meng ZY, Lun CB. Effects of selenium on fusarium growth and associated fermentation products and the relationship with chondrocyte viability. Biomed Environ Sci. (2017) 30:134-8. doi: 10.3967/bes2017.017

11. Yuan GQ, Yang H, Liang HQ, Zhou YH, Yang ZH, Zhou HZ. Breeding and fermentation condition optimization of a selenium-enriched yeast strain. Food Sci Technol. (2007) 5:42-5.

12. Kousha M, Yeganeh S, Amirkolaie AK. Effect of sodium selenite on the bacteria growth, selenium accumulation, and selenium biotransformation in Pediococcus acidilactici. Food Sci Biotechnol. (2017) 26:1-6. doi: 10.1007/s10068-017-0142-y

13. Ramalingam V, Rajaram R, Ananth S, Kaviyarasan M, Santhanam P. Development of thermostable amylase enzyme from Bacillus cereus for potential antibiofilm activity. Bioorg Chem. (2018) 77:14. doi: 10.1016/j.bioorg.2018.02.014

14. Bhagwat PK, Bhise KK, Bhuimbar MV, Dandge PBJES, Research P. Use of statistical experimental methods for optimization of collagenolytic protease production by Bacillus cereus strain SUK grown on fish scales. Environ Sci Pollut Res Int. (2018) 25:28226-36. doi: 10.1007/s11356-018-2859-4

15. Majumdar S, Goswami S, Keppen C, Rai SK, Mukherjee AK. Statistical optimization for improved production of fibrin(Ogen)olytic enzyme by Bacillus cereus strain FF01 and assessment of in vitro thrombolytic potential of protease enzyme. Biocatal Agricult Biotechnol. (2015) 4:1918. doi: 10.1016/j.bcab.2014.11.004

16. Scharek L, Altherr BJ, Tölke C, Schmidt MF. Influence of the probiotic Bacillus cereus var. toyoi on the intestinal immunity of piglets. Vet Immunol Immunopathol. (2007) 120:0-147. doi: 10.1016/j.vetimm.2007. 07.015
17. Zeng Y, Guo X, Zhou K, Liu S, Han X. Screening and identification of se-enriching lactic acid bacteria. Food Sci. (2015) 36:178-82.

18. Suhajda á, Hegóczki J, Janzsó B, Pais I, Vereczkey G. Preparation of selenium yeasts I. Preparation of selenium-enriched Saccharomyces cerevisiae. J Trace Elem Med Biol. (2000) 14:43-7. doi: 10.1016/S0946-672X(00)8 0022-X

19. Jin Zq, Zhang Bw, Li Pl. Selenium tolerance and enrichment in Bifidobacterium animalis. Food Sci. (2009) 30:184-7.

20. Congcong L, Yujie S, Zhiming H, Hongxu G, Xianyu D, Lianbing L. Screening of se-enriched probiotics and the optimization of se-enriched conditions. $J$ Food Sci Biotechnol. (2016) 35:667-71.

21. Hong Q, Jiaming Y, Feng J, Hongli Y, Weiming T. Se-enrichment characteristics of Enterobacter mori and its Se strengthening effect on corn grain. Trans Chin Soc Agricult Engin. (2018) 34:284-90.

22. Yang JP, Li J, Fan MT, Wei XY. Selenium-enriched lactic acid bacteria and evaluation of their bioactivities in vitro. Chin Inst Food Sci Technol. (2019) 19:171-82.

23. Xu Y, Lv JL, Zhang Y, Chang RY. Study on the enrichment of selenium by biotransformation in Lactobacillus. Shanxi Univer Sci Technol. (2018) 36:52-6.

24. Ying X, Shufang W, Fulian Y, Haixia Y, Jiaxuan H. Enhancement of Salt Stress on selenium-enrichment in Lactobacillus rhamnosus. Food Sci. (2020) 41:179-83.

25. Kessi J, Ramuz M, Wehrli E, Spycher M, Bachofen R. Reduction of selenite and detoxification of elemental selenium by the phototrophic bacterium Rhodospirillum rubrum. Appl Environ Microbiol. (1999) 65:473440. doi: 10.1128/AEM.65.11.4734-4740.1999

26. Van Fleet-Stalder V, Chasteen TG, Pickering IJ, George GN, Prince RC. Fate of selenate and selenite metabolized by Rhodobacter sphaeroides. Appl Environ Microbiol. (2000) 66:4849-53. doi: 10.1128/AEM.66.11.4849-48 53.2000

27. Roux M, Sarret G, Pignot-Paintrand I, Fontecave M, Coves J. Mobilization of selenite by Ralstonia metallidurans CH34. Appl Environ Microbiol. (2001) 67:769-73. doi: 10.1128/AEM.67.2.769-773.2001

28. Dungan RS, Yates SR, Frankenberger WT. Transformations of selenate and selenite by Stenotrophomonas maltophilia isolated from a seleniferous agricultural drainage pond sediment. Environ Microbiol. (2010) 5:28795. doi: 10.1046/j.1462-2920.2003.00410.x

Conflict of Interest: XCo and TY were employed by the company Enshi Se-Run Health Tech Development Co., Ltd.

The remaining authors declare that the research was conducted in the absence of any commercial or financial relationships that could be construed as a potential conflict of interest.

Copyright (C) 2020 Chen, Li, Cong, Yu, Zhu, Barba, Marszalek, Puchalski and Cheng. This is an open-access article distributed under the terms of the Creative Commons Attribution License (CC BY). The use, distribution or reproduction in other forums is permitted, provided the original author(s) and the copyright owner(s) are credited and that the original publication in this journal is cited, in accordance with accepted academic practice. No use, distribution or reproduction is permitted which does not comply with these terms. 